\title{
MUJER Y LITERATURA EN EL PERÚ DEL SIGLO XIX
}

\author{
WOMAN AND LITERATURE IN PERU IN THE 19TH CENTURY
}

Inmaculada Lergo Martín

Universidad de Sevilla

\section{RESUMEN:}

El siglo XIX fue una época de cambios constantes en la que las mujeres alzaron sus voces para reclamar su papel en la sociedad del momento. Este artículo pretende profundizar en el estudio de una serie de mujeres peruanas utilizaron sus obras para reivindicar valores y principios sociales. Entre estas autoras destaca Flora Tristán.

\section{Palabras claves:}

Perú, mujer, Flora Tristán.

\section{Abstract:}

The 19th century was a period of changes in which women raised their voices in order to demand their role in the society in that time. This article aims to go deeper into the study of several Peruvian women who used their works for reclaiming some social values and principles. Flora Tristán stands out from these writers.

\section{KEY WORD:}

Peru, woman, Flora Tristán. 
En 1924 Elvira Garcia García, con motivo de la Segunda Conferencia Panamericana de Mujeres celebrada en Lima, presenta a la comisión su libro La mujer peruana a través de los siglos: Serie historiada de estudios y observaciones. Y lo hace -dice a modo de justificación-, porque no se había escrito nada hasta el momento sobre la mujer peruana. Considera Elvira García que, en el pasado, aunque la mujer había sido siempre compañera de su esposo, de su hermano, de su amigo, etc., siempre había estado a la sombra y no se había reconocido su valor, y que en el presente a la mujer "se la conoce menos aún" y "se la juzga equivocadamente":

\section{Se la supone con actividad apenas, para gastarla en frivolidades sociales. Se le niegan sus derechos civiles y políticos, temiendo que, en el ejercicio de ellos, descienda de ser la eterna niña. Se limita su cultura intelectual, cortándole las alas muy temprano. Se sostiene que, con la ciencia rudimentaria que bebe en aquellos centros culturales, que la moda y las costumbres imponen, tien pensando que, es peligroso dejarla marchar muy allá" (6).}

Y siguen siendo necesarias ciertas reservas previas, de ahí que Emilio Gutiérrez de Quintanilla, que prologa y apoya un libro de estas características, haga su defensa de la mujer con ideas aún tremendamente conservadoras:

\section{Ella no es la espada; pero es el escudo que no ataca, y que en la defensa no conoce el castigo de la derrota, sino el glorioso y santo sacrificio del deber cumplico [...]. Su discreta reserva; excluye el desvarío del feministo, que propende dejar en vacancia, la función fisiológica, conservadora de la especie, para invadir órbitas absolutas y permanentemente masculinas [...]. Ni a la mujer, ni al hombre, le es lícito, dejar de ser los tipos armónicos, que hizo la naturaleza y definirlos en la bella frase de Napoleón Bonaparte: “La mujer es poesía: el hombre es prosa” (9-10).}

Escoge Elvira García para su obra a una serie de mujeres peruanas desde el Tahuantinsuyo hasta la actualidad. El tono es hagiográfico y conservador, pero es un primer paso. Muchos años después, en el prólogo a su obra Del silencio a la palabra. Mujeres peruanas en los siglos XIX-XX (1992) de Maritza Villavicencio volvemos a encontrar la misma queja: no hay estudios sobre la situación de la mujer peruana y por eso se presenta como un estudio de interés. Podemos decir que esto es así salvo por el texto Mujeres peruanas. El otro lado de la Historia de Sara Beatriz Guardia (1985). El siglo XIX en el mundo occidental es el de los nacionalismos, que en América sirvieron de base al proceso emancipador. Una vez calmados los conflictos internos que marcaron los primeros años de la República, que en el Perú se alargaron hasta mediados de siglo, se hacía necesario el control y centralización de todos los elementos de cohesión y definición de la nueva sociedad, entre ellos el papel de la mujer, que desde la Ilustración se estaba cuestionando y replanteando en el mundo occidental. Haciéndo eco de estas nuevas corrientes de pensamiento, los primeros gobernantes de la República parecían interesarse por ciertas cuestiones como, principalmente, la de prestar una mayor atención a la educación femenina y considerar su importancia para la sociedad. Sin embargo, desde la declaración de independencia en 1821 hasta el último tercio del siglo no se fue mucho más allá que de una declaración de intenciones.

\section{En el ámbito de la educación}

El pensamiento ilustrado hizo hincapié en la importancia de la educación para la mujer. Pero este interés incidía en la importancia de su papel en la familia, especialmente como madre, es decir, como educadora de los hijos y transmisora de una serie de valores. También consideró que había que impulsar su labor social, rechazando la costumbre de encerrar a las niñas en conventos donde eran educadas alejadas de la sociedad hasta el momento de su matrimonio. No hay que olvidar en este punto que la Ilustración fue muy crítica con la Iglesia, no tanto como portadora de una creencia religiosa que en sí no se cuestionaba, sino en cuanto a su estructura jerárquica y, sobre todo, al control efectivo ejercido sobre el poder político, sobre la sociedad en general e incluso sobre las conciencias. Una mujer con cierto nivel de educación y con la convicción de su importancia dentro de la familia y del ámbito social en el que se moviese estaría menos sometida a la presión de su confesor o director espiritual. Esta fue la causa real de las reivindicaciones de muchos ilustrados para fomentar y mejorar la educación femenina. Un documento muy interesante en este sentido es Importancia de la educación del bello sexo (1858) del clérigo liberal Francisco de Paula Rodríguez Vigil. Este clérigo apoyó la libertad de culto, fue defensor del matrimonio civil para los no católicos y se mostró contrario al celibato de los sacerdotes. Finalmente fue excomulgado por sus ideas.

González Vigil denuncia en este texto la situación de la mujer, considerando que era más esclava que compañera del hombre. Piensa que un pueblo civilizado se distingue de otro que no lo sea por el diferente trato que da a las mujeres. Sin embargo, esto no le impide valorar la importancia de su papel como madre y defender su sometimiento al marido o al padre. Aunque la explicación esté, como se ha dicho, en el intento de alejar de esta forma a la mujer de la fuerte influencia que la Iglesia ejercía sobre ella a través de los confesores, es significativo que incluso una mentalidad avanzada a su época como la de González Vigil no vaya más allá. Por supuesto tampoco la aceptaba en política: "lejos de nosotros el pensamiento de vindicar a la mujer el ejercicio de los derechos políticos, o de hacerla aparecer prestando su sufragio en las elecciones populares y magistraturas" (50-51).

Esta es la dirección en la que hay que encuadrar los primeros intentos en este siglo de educación para la mujer en el Perú. En los primeros años de la República se abrieron algunos colegios femeninos para la clase alta, con una educación digirida a su futuro de madre y esposa, y también a desempeñar un papel de cohesión social en el grupo al que pertenecise. La instrucción que recibían era por lo tanto diferente e inferior a la 
que se impartía en los colegios masculinos, y las clases eran de labores, cocina, dibujo, poesía, urbanidad y un poco de historia y literatura. No fue hasta 1872 cuando se estableció la obligatoriedad de la enseñanza primaria, aunque de nuevo fue más una intención sobre el papel que una realidad efectiva, pues sólo había en esos momentos dos colegios para mujeres regidos por monjas francesas.

Poco a poco, sin embargo, algunas mujeres comenzaron a luchar por el acceso a la educación superior. La primera mujer que entró en las aulas universitarias fue María Trinidad Enríquez, en 1874, en San Antonio Abad de Cuzco. Para ello, por lo extraordinario del hecho, necesitó una autorización especial. Aunque terminó sus estudios, no pudo llegar a graduarse, pues la tramitación del expediente fue tan largo que murió antes de que concluyese éste. Habrá que esperar a 1908 para que la universiadad abriera sus puerras a las mujeres, después de cuarenta años de luchas y reivindicaciones. Enríquez fundó un colegio de enseñanza femenina que impartía matemáticas, derecho, filosofía y lógica. Fue de fundamental importancia pues tras esta escuela surgirían otras más, como las de María Aragón de Rodo, Luisa Rausejour y Magdalena Chávez (Guardia: 52)

Estos hechos aislados no deben hacer pensar en que la posición de la mujer en la cultura había cambiado de la noche a la mañana, pero indica un momento de especial florecimiento y expansión de su papel en algún sector de la vida pública. En el último tercio del siglo XIX, algunas mujeres comenzaron a reivindicar un lugar en los círculos intelectuales y no sólo esto sino que fueron precisamente mujeres escritoras las que comenzaron a producir una serie de obras en prosa que iniciarán y caracterizarán el realismo y el naturalismo peruanos tras el auge de la bohemia romántica, y esto será, en el ámbito general de la historia de la literatura en el Perú, un hecho de considerable importancia.

\section{En el ámbito del trabajo}

Otro de los elementos de transformación de la situación la mujer en este siglo fue el del trabajo. Paralelamente a la expansión de la industria se produjo la aparición del proletariado, dentro del cual estaba la mujer, que se vio obligada a trabajar fuera de casa igual que el hombre. Las mujeres trabajaban en las fábricas en secciones y tareas destinadas a ellas y remuneradas por debajo de las de los hombres. Por otro lado, el miedo a la posible toma de conciencia de estas mujeres al salir del ámbito estrictamente familiar y poderse comunicar entre ellas se puede deducir del hecho de la prohibición expresa de hablar durante toda la jornada laboral. En el otro extremo de la escala social y también dentro de la burguesía se produjeron igualmente cambios significativos, principalmente tras la Guerra del Pacífico, debido al empobrecimiento del país. Dentro de estos grupos sociales las mujeres se dedicaron sobre todo a la enseñanza, pero también aumentaron las enfermeras y las que regentaban inmuebles para hacerlos rentar. Pero hay que tener en cuenta que lo habitual, en cualquier caso, era que la mujer, al contraer matrimonio, dejara los estudios o el trabajo pues era visto como una ofensa para el marido. Por otro lado, tampoco se le permitía ejercer ninguna actividad lucrativa sin la sutoización del marido. Para Maritza Villavicencio, todas estas circunstancias son la prueba de que el camino fue largo, nada fácil y cargado de contradicciones, y de que "en el Perú y más específicamente en Lima, muchas veces ocurre que lo que las mujeres ganan como trabajadoras lo pierden como mujeres, y viceversa" (149)

Con los conflictos armados surgieron también las llamadas "rabonas", mujeres que, en gran número, acompañaron a los hombres (padres, hermanos, esposos...) en el ejército y en las partidas que se formaron. El contundente testimonio que Flora Tristán nos deja de ellas en Peregrinaciones de una paria es el siguiente:

\section{forman una tropa considerable, preceden al ejército por el espacio de algunas horas para tener tiempo de conseguir víveres, cocinarlos y preparar todo en el albergue que deben ocupar. La partida de la vanguardia femenina permite enseguida juzgar los sufrimientos de estas desgraciadas y la vida de peligros y fatigas que llevan Las rabonas están armadas. Cargan sobre mulas las marmitas, las tiendas y, en fin, todo el bagaje. Arrastran en su séquito a una multitud de niños de toda edad. Hacen partir sus mulas al trote, las siguen corriendo, trepan así las altas montañas cubiertas de nieve y atraviesan los ríos a nado llevando uno y a veces dos hijos cubiertas des hijos sobre la espalda. Cuando llegan allugar que se les ha asignado, se ocupan primero en escoger el mejor sitio para acampar. En seguida descargan las mulas, arman
tienedas, amamantan y acuestan a los niños, encienden los fuegos y cocinan.}

Estas mujeres también atendían a los enfermos y heridos y enterraban a sus muertos. Se ignora cuántas fueron, cuántas murieron o quedaron heridas y su destino final pues la historia oficial se ha olvidado de ellas y sólo se nombran en una crónica de la guerra con Chile. También hubo "montoneras" luchando en partidas junto a los hombres, como María Olinda Reyes, apodada "Marta, La Cantinera", que tras una acción valerosa fue ascendida a capitana (Guardia: 62).

\section{En el ámbito político, intelectual y literario}

Los cambios que durante los siglos XVIII y XIX condujeron en Europa a la desestabilización de las estructuras políticas, sociales y económicas modificaron también las bases de pensamiento sobre la que se asentaba el modelo social y familiar imperante. Dentro de estas nuevas corrientes de pensamiento tendrán cabida el cuestionamiento del papel asignado a la mujer y la defensa de un nuevo estatus para ellas. En Hispanoamérica estos cambios se dieron en los últimos años de la Colonia y a partir de la Independencia. En el Perú, que contaba entonces con una clase alta 
dominante muy conservadora, la introducción de las nuevas corrientes de pensamiento se retrasó respecto a otras repúblicas independientes americanas, y los principales movimientos emancipadores se darán principalmente a partir de las primeras décadas del siglo XX.

Una personalidad que se adelantó a sus compañeras fue la de Flora Tristán. Hija de un peruano ilustre de rica familia y de madre francesa, nació en París y 1803. Su padre murió cuando era una niña y su madre no tuvo acceso a la fortuna familiar por carecer de los documentos que acreditaban su matrimonio. Muy joven comenzó a trabajar en una litografía y, a instancias de su madre, se casó con el dueño de la misma, André Chazal. Su matrimonio fue muy desgraciado. Flora Tristán lo abandonó, pero éste la persiguió durante años e incluso raptó a sus hijos. No pudo liberarse de él hasta que lo denunció por intento de incesto con su hija Aline y lo encarcelaron. Flora viajó a España, a Bilbao, donde se encontraba la parroquia y el sacerdote que había casado a sus padres, pero éste, a causa de la guerra de la Independencia con Francia que tenía lugrar en esos años, había huido con todos los documentos de su iglesia para salvarlos y no pudo localizarlo. Decidió entonces viajar al Perú para reclamar a su tío el reconocimiento oficial de la familia y la herencia que le correspondía, cosa que no consiguió. Era el año de 1834. De esta experiencia y de su actitud combativa ante la vida surgió su libro más conocido, Peregrinaciones de una paria (1839 en francés, $1^{\underline{a}}$ traducción al español 1946). Después se instaló en Londres, conoció a Robert Owen y sus ideas sobre el cooperativismo y el socialismo utópico, que enseguida adoptó como propias. Escribió otras muchas obras sobre todo dedicadas a la lucha social y a la emancipación femenina. Se la considera una precursora del socialismo científico, que en sus obras, como La unión obrera (1843), se adelantó a Carlos Marx -que tenía este libro en su biblioteca- y Federico Engels. En La emancipación de la mujer (1845) hizo un llamaniento a todas las mujeres del mundo para que defendiesen sus derechos políticos, sociales y económicos y con su unión hiciesen posible su logro. También abogó por la unión de las mujeres con los obreros para luchar jutos por una sociedad más justa. Tristán sería una desconocida en el Perú -sus libros se quemaron en la plaza de Arequipa y no se tradujeron al español hasta más de cien años después- hasta la recuperación de su figura hecha por Magda Portal en los años 40 del siglo XX.

Habla en su obra Flora Tristán de otras dos mujeres notables y luchadoras a las que tuvo ocasión de conocer: Dominga Gutiérrez y Francisca Zubiaga Bernales de Gamarra (1803-1835), conocida por La Mariscala. Ésta última era una mujer de carácter fuerte y pasional. De joven quiso ingresar en un convento, del que sus padres se vieron obligados a sacarla por los excesos que cometía en los ayunos y penitencias que se infligía. Después la llevaron al Cuzco, donde su personalidad y actuación cambiaron por completo y con la misma pasión que sintió antes por la religión se dedicó ahora a la vida social, al baile y las fiestas. Pronto conoció al que sería su esposo, el coronel Agustín Gamarra, que en el momento de conocerla estaba viudo y con un hijo. Se casaron el día antes de que Gamarra partiera para una misión como Jefe del Estado Mayor, y ella se quedó viviendo el Lima con su madre. Poco después su marido fue nombrado Prefecto del Cuzco y entonces Francisca se trasladó a vivir allí con su marido. Pronto se mostraría su verdadera personalidad y fuerza de carácter. No le interesaba la vida mundana y menos el papel destinado en ella a las mujeres, prefería una vida de acción, de ahí que aprendiese el manejo de las armas, a montar a caballo y todo lo que le permitiese una participación activa en los asuntos de su marido, al que impulsó continuamente para que llegase a ser presidente de la nación, cosa que ocurrió en 1929. Antes de esto tuvo lugar uno de los sucesos más comentados sobre su vida: conoció a Simón Bolívar cuando éste visitó el Cuzco, y se rumoreó que tuvieron un apasionado romance. Sea como fuere, su encuentro con Bolívar sirvió para afianzar su ambición de gloria y poder. El apoyo que brindó a su esposo no fue únicamente diplomático, sino que estuvo presente tanto en las reuniones políticas como en el campo de batalla. Sin embargo, su carácter, en muchas ocasiones soberbio e intolerante, le fue granjeando la enemistad popular, de forma que cuando cayó su marido en 1834 tras numerosos levantamientos en su contra, tuvo que huir del país. Murió al cabo de poco más de un año, el 8 de mayo de 1835. La tuberculosis y la agudización de los ataques epilépticos que siempre había sufrido terminaron con su vida.

Flora Tristán conoció a La Mariscala cuando ésta partía exiliada a Chile a bordo de un barco. Se entrevistó con ella en un par de ocasiones y dejó sus impresiones, como se ha dicho, en Peregrinaciones de una paria. Otra de las mujeres que aparecen en esta obra es Dominga Gutiérrez, protagonista de un episodio que dio lugar a un enorme escándalo en estos primeros años de la República. Lo comento aquí por ser ilustrativo de la situación de la mujer en estos momentos y al punto al que debían llegar aquellas que se resistían a ajustarse a tan estrechos márgenes. Dominga Gutiérrez era monja del monasterio de Santa Rosa, pero al no poder soportar más su situación y para poder escapar del monasterio, fue capaz de conseguir, con la ayuda de una esclava, el cadáver de una mujer joven, meterlo en el convento, vestirlo con sus hábitos, acostarlo en su cama y prenderle fuego para hacerlo pasar por ella. Así pudo huir, aunque finalmente todo se descubrió y hasta Roma intervino en el asunto. Cuando Flora Tristán tuvo acasión de hablar con ella, ésta le confesó amargamente que ni con su huida había conseguido ser libre:

¿Yo libre?... ¿y en qué país ha visto usted que una débil criatura, sobre quien cae el peso de un atroz prejuicio, sea libre? Aquí, en este salón ataviada con este lindo vestido de seda rosa ¡Dominga es siempre la monja de Santa Rosa! A fuerza de valor y constancia pude escapar de mi tumba. Pero el velo de lana que yo había 
elegido, está siempre sobre mi cabeza y me separa para siempre de este mundo. En vano he huido del claustro. Los gritos del pueblo me rechaza..

Peregrinaciones de una paria sacó a la luz la hipocresía de la sociedad del momento y ello le valió el desprecio de la clase dominante que la rechazó, quemó públicamente su libro y la obligó a salir del país. Antes del periodo que estamos considerando, la literatura peruana contaba con algunas obras fundamentales escritas por mujeres, como el anónimo Discurso en loor de la poesía o la Epístola a Belardo de Amarilis. Pero habían sido ejemplos aislados cuya autoría femenina fue incluso negada por algunos críticos. Va a surgir, sin embago, a partir de la década del 70 un grupo de mujeres con formación intelectual que dará lugar a una corriente más generalizada que propició la presencia femenina en un ámbito tradicionalmente masculino, la producción de obras de cierta proyección internacional, e incluso su aparición en algunas antologías que se hicieron eco de este proceso e incluyeron a poetisas dándoles un sitio en el Parnaso junto a los hombres.

Para Maritza Villavicencio, el hecho de que el desarrollo del pensamiento filosófico, científico y literario se produjese también fuera de los círculos intelectuales permitió a un cierto grupo de mujeres que habían logrado una buena preparación intelectual acercarse y participar en la vida intelectual y cultural del país. La mujer utulizó las tertulias, las veladas literarias, los clubes, los periódicos y las revistas como medio de introducirse y de participar junto a los hombres en este ámbito. $\mathrm{Y}$ apunta que "ello se debió, en alguna medida a la inversión económica que hicieron estas escritoras. Con el deseo de satisfacer necesidades espirituales y culturales propias y también de otras mujeres, sostuvieron materialmente periódicos y revistas, fundaron imprentas y mantuvieron las veladas de escritores, músicos y artistas de la época" (53-60).

Las primeras revistas escritas y dirigidas por mujeres fueron La Alborada (1875) y Álbum (1874). Hay muchas firmas en ellas pero las que participaban asiduamente fueron Juana Manuela Gorriti, Carolina Freyre, Juana Manuela Lazo de Eléspuru, Rosa Mercedes Riglos, Juana Rosa Amézaga, Leonor Sauri y Manuela Márquez. Las revistas no tenían contenido político ni social sino literario, artístico, y también algunos consejos de belleza y de cocina. Algunas mujeres también consiguieron dirigir revistas y periódicos importantes como El Perú ilustrado o El Centinela. Fuera como fuese, esto les permitió que se oyeran sus voces y sus opiniones sobre temas en los que no habían tenido cabida antes. Es interesante recordar que en el Perú fue la literatura escrita por mujeres la primera que atacó la esclavitud y defendió la igualdad entre los hombres.

El principal grupo de mujeres escritoras surgió, como se ha dicho, en los años 70 . El malestar social provocado por la Guerra del Pacífico (1879-1883) se vertió en protestas de los intelectuales, entre los que hubo voces femeninas. Las tertulias y las veladas literarias fueron el ámbito en que la mujer pudo hacer valer sus puntos de vista sin salir del hogar. A ellas invitaban a intelectuales y políticos, incluso enfrentados, con el ánimo de propiciar acuerdos. Fueron inauguradas por Juana Manuela Gorriti. La literatura se convirtió en el primer espacio para la defensa de la igualdad en el Peru y en una espita para la mujer en la expresión de sus anhelos y sentimientos. Mejor testimonio que estas brevísimas referencias lo ofrecen las propias escritoras. Clorinda Matto aprovecha una invitación del Ateneo de Buenos Aires en 1895 para hacer una denuncia y presentar a las "mujeres productoras que, no sólo dan hijos a la patria sino, prosperidad y gloria". Sin embargo, sabe que estas mujeres, en el medio en que se desenvuelven, "son verdaderas heroínas [...], luchan, día a día, hora tras hora, para producir el libro, el folleto, el periódico, encarnados en el ideal del progreso femenino" (Matto 1994: 254-255). Y ofrece un cumplida lista de escritoras hispanoamericanas y, sobre todo, peruanas.

Amalia Puga siente que la mujer está especialmente capacitada para las letras y lamenta que "antiguamente la ignorancia de los pueblos opusiera funestas preocupaciones, escrúpulos infundados, temores sin motivo, como otros tantos atajos, al genio de la mujer [...] para inspirarle aversión hacia el saber, y señaladamente hacia la literatura [...], se inventaron mil repugnantes anécdotas, tendentes todas a desacreditar los nombres de poetisas y escritoras que aparecían de tarde en tarde entre la masa grosera de los pueblos" (en Toro: 698-699). Sin embargo, tras la denuncia, hace el elogio de los tiempos presentes y de los hombres que la rodean, en un intento de ganar su apoyo y su aplauso:

ya han cambiado tanto las costumbres, se han ensanchado de tal modo las sociedades, viene extendiéndose de tan rápida manera la ilustración, que la mujer, por punto general ha cesado de ser la oscura sierva la sumisa esclava de ayer. [... en el día, lejos de ser rechazada, encuentra a su paso la mujer nobles corazones que en el dia, lejos de ser hue acrecienten su entusiasmo, robustos brazos que brinden apoyo, varoniles mano que la aplaudan; y por supuesto que casi han desaparecido los inconvenientes y la marcada oposición que de espinas llenaban su sendero, de ella sola depende ganarse lauros y rodear de honor su nombre, o perderse para siempre en las penumbras del olvido (701)

Y pasa a reflexionar sobre el importante papel que la mujer tiene en la literatura de la joven América, donde se han visto "nacer y desarrollarse sorprendentes talentos en el bello sexo", e invita a sus compatriotas a tributar "culto a las bellas letras, sea organizando pequeños círculos donde ensayar sus fuerzas, sea fundando amenas publicaciones con el propio objeto" (702-705). A pesar de todo lo dicho y como se ha visto, su trabajo se seguía viendo como un complemento de la maternidad y de su labor de esposas, cosa que defendieron casi con mayor fuerza las propias mujeres. Teresa González de Fanning escribía, bajo el pseudónimo de María de la Luz: 
Si... después de haber dado cumplimiento a sus deberes cotidianos, en vez de ir al teatro, a los paseos, o a reuniones dispendiosas, prefiere quedarse en casa escribiendo sus impresiones, o sus recuerdos y esperanzas, en tanto que vela el sueño de sus hijos o aguarda al esposo ausente, lejos de merecer censura, casi nos atreveríamos a decir que es acreedora de elogio y consideración de las persinos auiciosas. continus juiciosas... continua del genero humano, su fivolidad, sus gustos fútlles y aniñados, su apasionado culto a los extravagantes caprichos de la moda; y al mismo tiempo, se le condena con rara inconsecuencia, a no ocuparse de nada serio, so pena de incurrir en la nota pedante y bachillera (319)

Este razonamiento era una defensa ante la principal preocupación de los hombres: que abandonarían el hogar y sus obligaciones para con ellos y la familia. Escribía Mercedes Cabello:

\section{La reforma que quisiéramos ver realizada en la educación de la mujer, no la aleja de hogar doméstico; sino, por el contrario, le agrega un atractivo más, rodeándola del del alma, que esteriliza la vida (145-146)}

Otro punto de interés en cuanto a la posición de la mujer en el Perú de este siglo es el cuestionamiento de su participación en política. Todos los grupos de pensadores, desde los más conservadores a los más liberales, coincidieron en restringir la acción de las mujeres en este campo. Eso pensaban también, o defendían, muchas escritoras como Carolina Freyre de Jaimes que, siendo admiradora de Flora Tristán, consideraba que cometió el error de ser reformadora, y ve también como un desatino las peticiones de las feministas europeas en cuanto a tener voto y poder ser votadas para cargos públicos; o escritoras contestatarias como Matto de Turner que decía:

La mujer ha nacido para madre y debe ser toda ternura y sentimientos, porque el código que le rige es el corazón. Por eso pido para el varón el bullicio de la política, donde todos se engañan unos a otros, en medio de las serias genufexiones de la diplomacia y para la mujer elattar de la familia donde ella atiza el fuego sag de diplomacia, y para la mujer el altar de la familia, donde ella atiza el fuego sagrado y la Verdad [...]. Contemplar a la mujer con kepí, cartuchera al cinto y rifle al brazo causa risa y pena, mientras que la madre arrullando en sus brazos al hijo de su alma o implorando a Dios por los seres que ama, comunica paz, alegría y veneración (814).

\section{Y Mercedes Cabello de Carbonera:}

La política en sí es árida y no tiene un solo punto de contacto con la índole y e carácter femenil... ¿Qué alcanzaría la mujer el día que tuviera derechos políticos? Nada. Ningún consuelo para sus penas, ni una luz para su inteligencia, ni un sostén para su virtud (146)
Teresa González de Fanning fue quien captó la dimensión social del problema y dio los primeros pasos para el discurso feminista. El punto principal de discusión fue la educación y su oficio de escritoras. Todos estos testimonios confirman la apreciación de Villavicencio:

El discurso femenino fue tibio y se cuidó de no alterar lo que consideraba e fundamento biológico y social de la mujer. De allí que constantemente las escritoras, para defender su oficio, señalaran que sus actividades no se contradecian con sus responsabilidades de madres, hijas o esposas, las cuales reconocían como las principales. Muchos de sus temas poéticos estuvieron precisamente, inspirados en el hales. en ante los demás, descargando cualquier duda (59).

Estas contradicciones indican que el proceso no fue fácil. Mercedes Cabello de Carbonera terminó sus días sola en un sanatorio de enfermos mentales. También tuvo problemas Clorinda Matto. Salió exiliada después de haber sido quemado uno de sus libros y excomulgada.

Haciendo un brevísimo repaso por los logros de estas mujeres en el campo específico de la literatura, encontramos una serie de escritoras y de obras. En primer lugar hay que decir que por lo general, aunque hubo muchas poetas románticas, fueron las novelistas las que tuvieron una importancia crucial en la marcha de la literatura del Perú republicano, ya que fueron precisamente dos mujeres las iniciadoras de los movimientos realista y naturalista peruanos.

En primer lugar debemos destacar Aves sin nido (1889) de Clorinda Matto de Turner (1854-1909), que está considerada como una de las novelas fundadoras del realismo en toda Hispanoamérica. Su autora, tras una vida de infortunios, se trasladó a Arequipa en 1881, donde fue jefe de redacción del diario La Bolsa, desde el que alentó el patriotismo de sus compatriotas en la guerra contra Chile. Más tarde, en Lima, dirigió el semanario El Perú ilustrado y recibió una buena acogida por parte de los círculos literarios de la ciudad. Otras obras suyas son Índole (1890) y Herencia(1895). Lo que más destaca la crítica es el haber sido pionera en la denuncia de la situación de los indios. Además de su labor literaria participó activamente en política contra Piérola y al lado de Cáceres. Esto hizo que Piérola, al tomar el poder, la deportase a Buenos Aires. Pero en su paso por Chile, Argentina y España fue recogiendo el éxito y el reconocimiento generales. Murió en el exilio.

Otra de las grandes fue Mercedes Cabello de Carbonera, iniciadora del naturalismo con obras como Sacrificio y recompensa (Premio Ateneo de Lima en 1886), Blanca Sol (1889) o El conspirador(1892). También frecuentó las tertulias literarias y publicó diversos ensayos entre los que destaca La novela moderna (1892). Respecto a las poetas podemos 
destacar los siguientes nombres: Carolina Freyre de Jaimes, Justa García Robledo, Manuela Antonia Márquez, Carolina García Bambarén, Leonor Sauri, Manuela Varela de Vildozo, Felisa Moscoso de Chávez, Adriana Buendía, Manuela Villarán de Plasencia, La Baronesa de Wilson, Juana Rosa de Amézaga, Carmen Pontts, entre otras. Esta cumplida nómina es interesante pues la mujer era vista como asunto y como receptora de la poesía, no como agente de ella. Ahora sus poemas empiezan a ser publicados e incluso recogidos en alguna antología, como el Parnaso peruano (1871) de José Domingo Cortés, en el que leemos:

Si para los escritores no hai en América un teatro, ni un aplauso, ni una felicitación, para las mujeres apenas hai algo más que indiferencia. Por eso no es raro apenas encontrar alguna, muy de tarde en tarde, que cultive las letras, que haya escrito una página. Sin embargo el Perú, que en muchas cosas es excepcional, lo es también en ésta (775).

Cortés, que también publicará Poetisas americanas. Ramillete del bello sexo hispanoamericano (1875), defiende la poesía femenina afirmando que puede igualar e incluso superar a la de los hombres, comparación que se hacía necesaria en un mundo cultural donde lo masculino más que dominante era exclusivo. Entre los comentarios que les dedica están los siguientes: "En las filas de los poetas peruanos en que forman no pocas inspiradas mujeres tiene conquistado ya un puesto honrosa la señorita Manuela Antonia Márquez, hermana del conocido literato don José Arnaldo Márquez" (403) de Justa García Robledo dice que el lector verá que no exagera ni hace "una galantería a la poetisa al sostener que es una de las joyas del Parnaso Peruano" (280); sobre las composiciones de Leonor Sauri opina que "no necesitan de elogios, ni de personas que encarezcan su mérito. Ellas solas se recomiendan" (117-118). Pero también procura, junto con las alabanzas a su poesía, no descuidar el decir que son todas mujeres honrosísimas dedicadas a las tareas del hogar o a la devoción religiosa: "La vida de esta poetisa -dice de Manuela Villarán- debe rastrearse en el hogar, al lado de sus hijos, consagrada al culto de la simpática religión del deber y de la familia" (785); "se notó desde sus primeros años en ella -Justa García Robledo- una inclinación muy marcada por el recogimiento y la piedad" (279).

La muestra de poesía escrita por mujeres que aparece en esta antología, siendo dispar en su calidad literaria, ofrece una estética y logros similares a la que se ofrece de los poetas masculinos. La temática fundamentalmente es amorosa, patriótica o de idealización de la naturaleza. Lo interesante, en definitiva, es dejar constancia de estos primeros logros, valorar su importancia y apreciar el tesón, esfuerzo y valentía que supusieron para sus protagonistas y para los que las apoyaron en su lucha.

\section{REFERENCIAS BIBLIOGRAFICAS}

Basadre, J., "Prólogo" en Peregrinaciones de una paria, Lima, Cultura Antártica, pp. V-XXIII, 1946

Cabello de Carbonera, M., “Influencia de la mujer en la civilización”, Álbum (1874)

Cortés, J. D., Parnaso peruano, Valparaíso, Albión de Cox y Taylor, 1871.

Guardia, S. B., Mujeres peruanas. El otro lado de la Historia, Lima, Humboldt, 1985.

González de Fanning, T., “Las literatas”, El Correo del Perú, 40 (1º de octubre de 1876).

González Vigil, F. de P., Importancia de la educación del bello sexo, Lima, Instituto Nacional de Cultura, 1976.

Gutiérrez de Quintanilla, E., “Prólogo” a García García, Elvira, La mujer peruana a través de los siglos: serie historiada de estudios y observaciones, Lima, s. e., 1924.

----, “Las obreras del pensamiento en la América del Sud. (Lectura hecha por la autora en el Ateneo de Buenos Aires, el 14 de diciembre de 1895)", en Rotker, Sunana (ed.), Ensayistas de nuestra América, t. II. De Bello a

González Prada, Buenos Aires, Losada, 1994.

Matto de Turner, C., “Luz entre sombras. Estudio filosófico-moral para las madres de familia”, El Perú ilustrado, 81 (1889).

Portal, M. [et al.], Flora Tristán: una reserva de utopía, [Perú], Centro de la Mujer Peruana Flora Tristán, 1985.

Puga de Losada, A., “Discurso de incorporación como socia activa al Ateneo de Lima”, publicado en El Perú Ilustrado, cit. en Toro Montalvo, César: Historia de la literatura peruana, tomos VI, Lima, A.F.A., bajo el título de "La literatura en la mujer por Amalia Puga de Losada", pp. 697-709, 1996

Tristán, F., Peregrinaciones de una paria, Lima, Cultura Antártica, 1946.

Villavicencio, M., Del silencio a la palabra. Mujeres peruanas en los siglos XIX - XX, Lima, Flora Tristán, 1992. 